\title{
Pneumothorax after Brachial Plexus Block Guided by Ultrasonography: Case Report
}

I would like to thank the editor for the space to comment on the report by Mandin et al. ${ }^{1}$ I read it with interest and some questions occurred to me, raising pertinent comments.

\section{Question 1}

What was the puncture level and direction?

Comment: Winnie ${ }^{2}$ provided significant anatomical contribution for limb block management. Notably, the puncture direction described for interscalene (ISC) block is almost perpendicular to the skin, which facilitates iatrogenic damage to the brachial plexus and spinal tissue. Therefore, depending on patient's constitution, insertion deeper than $2.5 \mathrm{~cm}$ is not recommended ${ }^{3}$.

\section{Question 2}

Was the described click coming from the neurovascular sheath (NVS) or parietal pleura (PP)?

Comment: If the click came from parietal pleura puncture, it may well have resulted in sudden and fleeting pain, followed by cough.

\section{Question 3}

Twenty milliliters of Ropivacaine (Rp) $0.5 \%$ administered via ISC route under ultrasound guidance should clearly identify the involvement of trunks.

Comment: One of the ultrasound advantages in regional anesthesia in such cases is the reduced volume of anesthetic in real time. The completion of more than $20 \mathrm{~mL}$ of $\mathrm{Rp} 0.5 \%$ via axillary route motivated me to wonder about two things: a) ISC volume has not satisfactorily reached the plexus trunks, partially disappeared in the pleural space between both pleurae by the PP puncture and provoked insidious pneumothorax; b) the access to BNV was partial, with ectopic spread of the anesthetic, which potentially results in partial blockade. To ensure the blockade effectiveness, it was supplemented via axillary route.

\section{Question 4}

Why was the anteroposterior X-ray in Image 1 not complete, showing both lung fields?
Comment: X-ray chest showing both lung fields is more instructive, especially when there is suspicion of pneumothorax and/or diaphragmatic paresis of the target-lung field. In any case, there is a noticeable lung displacement from the costal wall to the right in the X-ray, which shows a small pneumothorax. Although the diaphragm is usually more elevated on the right side when compared to the contralateral side, due to liver volume, the diaphragmatic paresis discussed in the case report should be visualized in the X-ray. The arrows mentioned ${ }^{1}$, albeit not visible, should be in contrasted color (white or light yellow) against the dark image of the X-ray.

\section{Question 5}

Although there is no comment on postoperative analgesia (PO)-probably this was not the goal-, the patients in the postoperative period experienced pain with chest tube!

Comment: In the report, there is no evidence of pain and analgesia related to the surgery; however, chest tubes are very painful, especially during inspiration, which was not mentioned. The only report was the following: "The patient evolved with improved dyspnea, with drain functioning with oscillation. During hospitalization, there was need for repositioning the drain and use of continuous aspiration" 1 . Delayed chest drains cause pain and affect breathing. I believe the painful consequences of drain were not pertinent to the case.

Karls Otto Geier Hospital São Lucas da Pontifícia Universidade Católica/RS; Hospital Municipal de Pronto Socorro de Porto Alegre/RS, Brasil

\section{References}

1. Mandim BLS, Alves RR, Almeida R et al. - Pneumotórax pósbloqueio de plexo braquial guiado por ultrassonografia: relato de caso. Rev Bras Anestesiol. 2012;62:5:741-747.

2. Winnie AP - Interscalene brachial plexus block. Anesth Analg. 1970;49(3):455-466.

3. Sardesai $A M$, Patel $R$, Denny $N$ et al. - Interscalene brachial plexus block: can the risk of entering the spinal canal be reduced? Anesthesiology. 2006;105:9-15. 